\title{
Determinants of Aspirin Metabolism in Healthy Men and Women: Effects of Dietary Inducers of UDP-Glucuronosyltransferases
}

\author{
Sandi L. Navarro a, b Misty R. Saracino ${ }^{a}$ Karen W. Makar ${ }^{a}$ \\ Sushma S. Thomas ${ }^{\mathrm{a}}$ Lin Li $^{\mathrm{a}} \quad$ Yingye Zheng $^{\mathrm{a}}$ Lisa Levy $^{\mathrm{a}}$ \\ Yvonne Schwarz ${ }^{a}$ Jeannette Bigler ${ }^{a}$ John D. Potter ${ }^{a}$ \\ Johanna W. Lampe ${ }^{a, b}$ \\ ${ }^{a}$ Fred Hutchinson Cancer Research Center and ${ }^{b}$ Interdisciplinary Graduate Program in Nutritional \\ Sciences, Department of Epidemiology, University of Washington, Seattle, Wash., USA
}

\section{Key Words}

Aspirin • Citrus • Cruciferous vegetables • Glucuronidation • Soy •

UDP-glucuronosyltransferases

\begin{abstract}
Background/Aims: Interindividual variation in aspirin (ASA) metabolism is attributed to concomitant use of drugs or alcohol, urine $\mathrm{pH}$, ethnicity, sex, and genetic variants in UDP-glucuronosyltransferases (UGT). Little is known about the effects of diet. Methods: We evaluated crosssectionally whether urinary excretion of ASA and its metabolites [salicylic acid (SA), salicyluric acid (SUA) phenolic glucuronide (SUAPG), salicylic acid acyl glucuronide (SAAG) and salicylic acid phenolic glucuronide (SAPG)] differed by UGT1A6 genotype and dietary factors shown to induce UGT. Following oral treatment with $650 \mathrm{mg}$ ASA, urine was collected over $8 \mathrm{~h}$ in 264 men and 264 women (21-45 years old). Results: There were statistically significant differences in metabolites excreted between sexes and ethnicities. Men excreted more SUA; women more ASA ( $p=0.03)$, SA, SAAG and SAPG ( $p \leq 0.001$ for all). Compared to Caucasians, Asians excreted more ASA, SA and SAAG, and less SUA and SUAPG ( $p \leq 0.03$ for all); African-Americans excreted more SAAG and SAPG and less SUA ( $p \leq 0.04)$. There was no effect of UGT1A6 genotypes. Increased ASA and decreased SUAPG excretion was observed with increased servings of vegetables $(p=0.008)$, specifically crucifers $(p=0.05)$. Conclusion: Diet may influence the pharmacokinetics of ASA, but effects may be through modulation of glycine conjugation rather than glucuronidation.
\end{abstract}




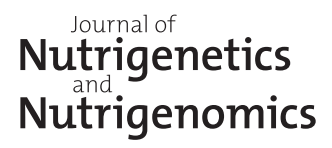

\begin{tabular}{l|l}
\hline J Nutrigenet Nutrigenomics 2011;4:110-118 \\
\hline DOI: 10.1159/000327782 \\
Published online: May 28, 2011 & ○ 2011 S. Karger AG, Basel \\
\hline Navarro et al.: Determinants of Aspirin Metabolism &
\end{tabular}

\section{Introduction}

High interindividual variation in aspirin (ASA) metabolism and therapeutic response has been observed and is attributed to many factors, including concomitant use of other drugs or alcohol [1], urinary pH [2], sex [3], ethnicity [4], and variants in metabolizing enzymes involved in glucuronidation of ASA metabolites [i.e., UDP-glucuronosyltransferases (UGT)] $[5,6]$. The present analysis extends previous work to include an evaluation of the influence of dietary factors on ASA metabolism.

ASA (acetyl salicylic acid) is rapidly deacetylated to salicylic acid (SA) and then further metabolized. Hydroxylation of SA, a minor metabolic pathway, is accomplished by cytochrome $\mathrm{P}_{450}$ (CYP) enzymes to form gentistic acid, whereas UGT enzymes convert SA to glucuronides [salicyl acid phenolic glucuronide (SAPG) and salicyl acid acyl glucuronide (SAAG)], and salicyluric acid (SUA) phenolic glucuronide (SUAPG) after glycine conjugation [7]. Glucuronides represent a substantial proportion of ASA metabolites [7]. Several genetic variants have been identified in the UGT1A family that result in altered enzyme activity [8-10]. UGT1A6* 2 contains two missense mutations in exon 1 that result in T181A and R184S amino-acid substitutions [8]. These substitutions result in a slower metabolism of some phenolic compounds and an increase in others compared to the wild type (UGT1A6*1) $[8,11$, 12]. Studies have reported an allele frequency of $\sim 17-33 \%$ for this variant in predominantly Caucasian populations $[8,13]$. We have previously shown that the protective effects of ASA are modified by the UGT1A6 genotype. Among ASA users, only individuals carrying UGT1A6 variant alleles had a reduced risk of colorectal adenomas [14]. Based on these and other studies reporting similar results, we hypothesized that slow glucuronidation in carriers of the UGT1A6 variant alleles would lead to increased salicylate levels, the main pharmacologically active compound in ASA [15].

Dietary constituents of citrus fruits (e.g., quercetin) [16], cruciferous vegetables (e.g., isothiocyanates and indoles) [17], and soy (e.g., genistein) [18] have been shown to induce UGT expression, both in animal models and in humans [18]. Higher intakes may alter UGT activity and affect the ratio of ASA metabolites excreted. Most evidence suggests that up-regulation of phase 2 enzymes by these phytochemicals occurs through interaction with the cytoplasmic-anchoring protein Keap 1 (Kelch-like ECH-associated protein 1) and the human transcription factor $\mathrm{Nfe} 2 \mathrm{l} 2$ (nuclear factor erythroid-derived related-factor 2-like 2; also known as Nrf2) via the antioxidant response element [16, 19]. Indoles in crucifers are thought to act through the xenobiotic response element via the aryl hydrocarbon receptor, which induces both phase 1 and 2 enzymes [20, 21]. To our knowledge, no human studies have addressed the possibility that consumption of foods rich in bioactive phytochemicals may alter the relationship between polymorphisms and the metabolism of ASA. The aims of this study were to identify overall effects of UGT1A6 genotypes and to determine whether the pattern of glucuronide metabolites of ASA differs by dietary factors, particularly soy, citrus, and cruciferous vegetable intake.

\section{Methods}

\section{Study Participants}

Healthy, non-smoking men and women (aged 21-45 years) were recruited from the greater Seattle area between June 2003 and March 2007. Methods of recruitment and exclusion criteria have been described elsewhere [16]. Briefly, exclusion criteria included factors known to influence biotransformation enzyme induction, e.g., current use of over-the-counter (including ASA) or prescription drugs, alcohol intake $>2$ drinks/day, or regular exposure to passive smoke or organic solvents. Participants were asked to discontinue use of all multivitamins and dietary supplements 1 week prior to their participation in the 
study. Data from this study were pooled with another sample collected from a separate study (Aspirin, UGT1A6 Genotype and Colon Gene Expression) for a total of 615 individuals. We excluded 87 individuals: 31 with a UGT1A6 genotype other than a combination of * 1 and * 2 alleles; 48 with inaccurate urine collection (defined by $<7$ or $>9$ h); 6 with extreme body mass index (BMI; defined as $<15$ or $>50$ ), and 2 individuals with $<1 \%$ total recovery, probably due to failure to consume the ASA, for a final sample of 528 . Screening and study protocols were the same for both studies [16]. The Institutional Review Board at the Fred Hutchinson Cancer Research Center approved the study, and informed consent was obtained from all participants.

\section{Data and Biologic Sample Collection}

For this cross-sectional study, participants completed a food frequency questionnaire (FFQ) reporting their dietary intake within the past 3 months and a health and demographic questionnaire. FFQs with incomplete information, or data that suggested biologically implausible daily energy intakes of $<600$ or $>4,000 \mathrm{kcal}$ for women or $<800$ or $>5,000 \mathrm{kcal}$ for men were excluded [a modification from ref. 22]. FFQ data were thus available for 481 participants. FFQ details have been described elsewhere [16]. FFQ line items included in each category were: broccoli, cauliflower, cabbage, Brussels sprouts, and coleslaw, for cruciferous vegetables; oranges and orange juice, grapefruits and grapefruit juice, and tangerines, for citrus; and tofu, tempeh, products such as soy hot dogs and burgers, tofu cheese, and miso soup for soy. Body weight and height were measured, and blood was drawn in the morning after a 12-hour overnight fast for UGT genotyping $[13,14]$ and standard blood chemistry panel.

Participants were instructed to avoid consumption of all food and beverages $2 \mathrm{~h}$ prior to ASA dosing in the evening before bed; void; take an oral dose of $650 \mathrm{mg}$ ASA (Bayer Inc., Pittsburgh, Pa., USA), and collect all urine for the next $8 \mathrm{~h}$ (in most instances, this was a single void after waking). Urine specimens were stored at $4^{\circ} \mathrm{C}$ until delivery to the Fred Hutchinson Cancer Research Center the morning following collection. Total volume and initial $\mathrm{pH}$ of urine samples were recorded prior to adjustment (to $\mathrm{pH} 3-4$ ) with concentrated phosphoric acid to minimize (SAAG) degradation [23], aliquoted, and stored at $-80^{\circ} \mathrm{C}$.

\section{Urinary ASA Metabolite Measurements}

Urinary metabolites and their glucuronidated conjugates were analyzed by high-performance liquid chromatography [6]. The limits of quantification in urine samples were $2 \mathrm{pmol} / \mathrm{l}$ for SA, SUA, SAPG, SAAG, SUAPG and $1 \mathrm{pmol} / \mathrm{l}$ for ASA. The coefficients of variation for ASA and its metabolites in quality control urine samples were 10.1\% (ASA), 12.2\% (SA), 2.8\% (SUA), 5.3\% (SAAG), 17.2\% (SAPG), and 11.0\% (SUAPG).

Urinary ASA and its metabolites ( $\mu \mathrm{mol})$ were calculated as [total volume of collected urine over $8 \mathrm{~h}$ $(\mathrm{ml})] \times[$ concentration of each metabolite $(\mu \mathrm{mol} / \mathrm{ml})]$. Total amounts of ASA and its metabolites (TOTAL) were calculated by summing the amounts in the urine (ASA + SA + SUA + SAAG + SAPG + SUAPG). Glucuronide was the sum of SAAG, SAPG, and SUAPG. The proportion of each metabolite, as a percentage of all the excreted urinary metabolites, was calculated by dividing the amount of each metabolite by the total amount of excreted urinary metabolites and multiplying by 100 (e.g., ASA/TOTAL, SA/TOTAL, SUA/TOTAL, SAAG/TOTAL, SAPG/TOTAL, SUAPG/TOTAL, and glucuronide/TOTAL × 100).

\section{Statistical Analysis}

Urinary ASA metabolites were log transformed for normality and to reduce the contribution of outliers. Multiple linear regression was used to assess the association of habitual dietary intake and ASA metabolism. Analyses were adjusted a priori for UGT1A6 genotype and sex. Variables for cruciferous vegetables, citrus, and soy were analyzed as tertiles of intake, with equal numbers of individuals within each tertile, and examined both as categorical (highest and medium tertile vs. lowest tertile separately) and as continuous variables for tests for trend (comparison of mean values across each tertile). Total protein, fruit, vegetable, and energy were analyzed as continuous variables. The following covariates were assessed as potential confounders or predictors in all regression models: age, body weight, height, BMI, urine volume and $\mathrm{pH}$, energy intake and the interactions between sex, ethnicity, and UGT1A6 and UGT1A1 genotypes. Predictors were included in all models if the $\mathrm{p}$ value for the test of the regression coefficient for the variable term was equal to 0 and statistically significant $(\mathrm{p}<0.05)$ for any metabolite. Confounders were included if their addition to the regression model changed the main effect estimates by $\geq 10 \%$. Statistical tests, performed using StataSE 11.0 (StataCorp), were two sided, with $\mathrm{p}<0.05$ considered statistically significant. 
Table 1. Characteristics of the study participants stratified by sex and UGT1A6 genotypes

\begin{tabular}{|c|c|c|c|c|c|c|}
\hline & \multicolumn{6}{|c|}{ UGT1A6 genotype } \\
\hline & \multicolumn{3}{|c|}{ men $(n=264)$} & \multicolumn{3}{|c|}{ women $(n=264)$} \\
\hline & $\begin{array}{l}{ }^{*} 1 /{ }^{*} 1 \\
(n=129)\end{array}$ & $\begin{array}{l}{ }^{*} 1 /{ }^{*} 2 \\
(\mathrm{n}=111)\end{array}$ & $\begin{array}{l}{ }^{*} 2 /{ }^{*} 2 \\
(\mathrm{n}=24)\end{array}$ & $\begin{array}{l}{ }^{*} 1 /{ }^{*} 1 \\
(\mathrm{n}=136)\end{array}$ & $\begin{array}{l}{ }^{*} 1 /{ }^{*} 2 \\
(n=103)\end{array}$ & $\begin{array}{l}{ }^{*} 2 /{ }^{*} 2 \\
(n=25)\end{array}$ \\
\hline Age, years & $31(7.5)$ & $31(7.4)$ & $29(5.7)$ & $30(7.4)$ & $31(6.9)$ & $28(6.6)$ \\
\hline Height, cm & $179(8.0)$ & $178(7.1)$ & $179(7.5)$ & $165(7.3)$ & $167(7.3)$ & $165(7.8)$ \\
\hline Weight, kg & $81(15.7)$ & $82(15.9)$ & $81(17.5)$ & $66(13.1)$ & $69(16.9)$ & $70(13.8)$ \\
\hline BMI & $25(4.3)$ & $26(5.0)$ & $26(5.2)$ & $24(4.1)$ & $25(5.9)$ & $26(4.7)$ \\
\hline \multicolumn{7}{|l|}{$U G T 1 A 1^{\mathrm{a}}$} \\
\hline${ }^{*} 1 /{ }^{*} 1(\mathrm{n}=252)$ & $109(87)$ & $14(11)$ & $2(2)$ & $113(89)$ & $14(11)$ & 0 \\
\hline${ }^{*} 1 /{ }^{*} 28(\mathrm{n}=221)$ & $15(14)$ & $93(84)$ & $6(5)$ & $17(16)$ & $87(81)$ & $3(3)$ \\
\hline${ }^{*} 28 / * 28(\mathrm{n}=54)$ & $4(17)$ & $4(17)$ & $16(67)$ & $6(20)$ & $2(7)$ & $22(73)$ \\
\hline \multicolumn{7}{|l|}{ Ethnicity } \\
\hline Caucasian & $79(46)$ & $78(45)$ & $16(9)$ & $84(47)$ & $73(41)$ & $22(13)$ \\
\hline Asian & $19(59)$ & $8(25)$ & $5(16)$ & $27(64)$ & $13(20)$ & $2(3)$ \\
\hline African-American & $11(65)$ & $5(29)$ & $1(6)$ & $7(54)$ & $6(46)$ & 0 \\
\hline Other & $20(48)$ & $20(48)$ & $2(5)$ & $18(60)$ & $11(37)$ & $1(3)$ \\
\hline
\end{tabular}

There were no statistically significant differences across UGT1A6 genotypes regarding sex at $\mathrm{p}<0.05$ ( $\chi^{2}$ for categorical variables; ANOVA for continuous variables). Means ( $\pm S D$ ) are shown for age, height, weight and BMI, and n (\%) for UGT1A1 (proportions may not total 100\% due to rounding). Other ethnicity includes unknown/refused $(\mathrm{n}=25)$, mixed races $(\mathrm{n}=42)$, American Indians $(\mathrm{n}=2)$, and Pacific Islanders $(n=3)$.

a There is 1 missing male UGT1A1 genotype. Percentages reflect the distribution of UGT1A6 genotype within UGT1A1 genotype and race for each sex.

\section{Results}

There were no statistically significant differences in the demographic characteristics of the 528 individuals by UGT1A6 genotype and sex (table 1). Distribution of UGT1A6 genotypes was $50 \%(\mathrm{n}=265)$ homozygous wild type $\left({ }^{*} 1 /{ }^{*} 1\right), 41 \%(\mathrm{n}=214)$ heterozygous $\left({ }^{*} 1 /{ }^{*} 2\right)$, and $9 \%(\mathrm{n}=49)$ homozygous variant $\left({ }^{*} 2{ }^{*} 2\right)$ and they were in high linkage disequilibrium with $U G T 1 A 1$ genotypes, as has been illustrated elsewhere [13]. Of those with a $U G T 1 A 6^{*} 1 /{ }^{*} 1$ genotype, $84 \%$ were also $U G T 1 A 1^{*} 1{ }^{*} 1 ; 84 \%$ who were $U G T 1 A 6^{*} 1{ }^{*} 2$ were $U G T 1 A 1^{*} 1{ }^{*} 28$, and $78 \%$ who were $U G T 1 A 6^{*} 2 /{ }^{*} 2$ were $U G T 1 A 6^{*} 28 /{ }^{*} 28$.

Table 2 presents mean percents of metabolites excreted by sex and ethnicity. Statistically significant differences in the ratios of metabolites excreted were observed between sexes and between ethnic groups. Men excreted more SUA (3\%; $p<0.001)$, and women excreted more ASA (9\%; $p=0.03)$, SA (11\%; $\mathrm{p}=0.001)$, and the glucuronides SAAG and SAPG ( 9 and $10 \%$, respectively; $\mathrm{p} \leq 0.001$ for both). Compared to Caucasians: Asians excreted statistically significantly more ASA $(21 \%$; $\mathrm{p}=0.002)$, SA $(23 \%$; $\mathrm{p}<0.0001)$ and SAAG, $(11 \%$; $\mathrm{p}=0.03)$ and less SUA (4\%; $\mathrm{p}<0.0001)$ and SUAPG (35\%; $\mathrm{p} \leq 0.002)$, and African-Americans excreted more SAAG and SAPG (16\%; $p<0.001)$, and less SUA (6\%; $p \leq 0.04)$. The interaction term for sex*ethnicity was statistically significant for SUAPG $(\mathrm{p}=0.006)$ and SUA $(\mathrm{p}=0.04)$, with men excreting more of both metabolites than women for all races except African-Americans (data not shown). Excretion of urinary ASA metabolites did not differ statistically significantly between UGT1A6 genotypes (data not shown). 
Table 2. Urinary ASA metabolites excreted by sex and ethnicity

\begin{tabular}{|c|c|c|c|c|c|c|}
\hline & \multicolumn{6}{|c|}{ ASA metabolite recovered, $\%$} \\
\hline & ASA & SA & SAAG & SAPG & SUA & SUAPG \\
\hline \multicolumn{7}{|l|}{ Sex } \\
\hline Male & $15.4(0.4)$ & $16.8(0.4)$ & $33.9(0.1)$ & $38.1(0.6)$ & $84.7(0.3)$ & $20.2(1.5)$ \\
\hline Female & $16.9^{\mathrm{a}}(0.4)$ & $19.3^{\mathrm{a}}(0.4)$ & $37.1^{\mathrm{a}}(0.1)$ & $41.4^{\mathrm{a}}(0.6)$ & $82.4^{\mathrm{a}}(0.3)$ & $17.2(1.3)$ \\
\hline \multicolumn{7}{|l|}{ Ethnicity } \\
\hline Caucasian & $15.5(0.3)$ & $17.2(0.4)$ & $34.2(1.1)$ & $38.5(0.5)$ & $84.5(0.3)$ & $19.4(1.2)$ \\
\hline Asian & $18.6^{\mathrm{b}}(0.9)$ & $21.3^{\mathrm{b}}(0.1)$ & $37.7^{\mathrm{b}}(1.1)$ & $42.3(1.3)$ & $81.4^{\mathrm{b}}(0.6)$ & $14.6^{\mathrm{b}}(2.0)$ \\
\hline African-American & $16.0(1.2)$ & $19.3(0.2)$ & $40.6^{\mathrm{b}}(1.9)$ & $45.2^{\mathrm{b}}(2.1)$ & $79.4^{\mathrm{b}}(0.9)$ & $14.5(3.2)$ \\
\hline Other & $16.6(0.9)$ & $18.6(0.1)$ & $37.2(1.1)$ & $41.2(1.2)$ & $82.5(0.6)$ & $21.8(3.2)$ \\
\hline
\end{tabular}

All statistical analyses were performed on log-transformed percents of metabolite recovered (metabolite/total recovered $\times 100$ ) adjusted for BMI, age, UGT1A6 genotype, sex, ethnicity, and urinary volume and $\mathrm{pH}$. Other ethnicity includes unknown/refused $(\mathrm{n}=25)$, mixed races $(\mathrm{n}=42)$, American Indians $(\mathrm{n}=2)$, and Pacific Islanders $(\mathrm{n}=3) .{ }^{\mathrm{a}} \mathrm{p}<0.05$ vs. male; ${ }^{\mathrm{b}} \mathrm{p}<0.05$ vs. Caucasians (concentrations do not total $100 \%$ due to adjustment).

Statistically significantly increased ASA and decreased SUAPG excretion was observed with more servings of total vegetables $(>2.5$ servings of vegetables/day compared to $<1 ; \mathrm{p}=$ 0.008 for both), and a trend toward statistical significance with intake of cruciferous vegetables specifically ( $\mathrm{p}=0.05$ for both; table 3 ). Although the overall trend in SA excretion with increasing crucifer consumption was not statistically significant, individuals consuming 1-2.5 servings/week excreted significantly more SA than those consuming $<1$ serving/week $(\mathrm{p}=0.046)$. Similarly, the overall trend in SUA was not statistically significant; however, individuals consuming 1-2.5 servings/week secreted significantly less SUA than individuals consuming $<1$ serving/week ( $\mathrm{p}<0.03$ ). There were no statistically significant differences in metabolite excretion by total protein, total fruit, energy, soy, or citrus fruit intake.

Mixed linear regression models including sex, ethnicity, BMI, age, UGT1A6 genotype, and urine volume and $\mathrm{pH}$ accounted for $\sim 18 \%$ ( $\mathrm{R}^{2}$ values) of the variability in ASA metabolism, with sex and ethnicity accounting for the majority (up to $\sim 13 \%$ ), and the remaining factors contributing $<3-5 \%$ combined. The inclusion of dietary data in the model (total energy, protein, fruit and vegetables) did not add substantially to the variability $(<2 \%)$.

\section{Discussion}

Differences in the therapeutic response to ASA have long been recognized. The rate of metabolism and excretion of ASA and its metabolites likely plays a role in determining these differences. In this study, we found that women excreted more ASA, SA, and the glucuronides SAAG and SAPG compared to men, whereas men excreted more SUA; nonetheless, SUA was the major metabolite excreted by all individuals. This observation was driven mainly by higher excretion of ASA among Asian women, higher glucuronides (SAAG and SAPG) by AfricanAmerican women, and higher SUA by Caucasian men. Although the magnitude of the differences between the sexes was small, the ethnic differences were greater and are potentially of clinical relevance. Our results are in agreement with previous studies reporting sex differences in ASA metabolism and disposition [24, 25]. These differences in drug metabolism have 
Table 3. Urinary ASA excretion by tertiles of intake for crucifers, citrus and soy

\begin{tabular}{|c|c|c|c|c|c|c|}
\hline \multirow{2}{*}{$\begin{array}{l}\text { Dietary component } \\
\text { (servings) }\end{array}$} & \multicolumn{6}{|c|}{ ASA metabolite recovered, \% } \\
\hline & ASA & SA & SAAG & SAPG & SUA & SUAPG \\
\hline \multicolumn{7}{|l|}{ Total vegetables } \\
\hline Low (<7/day) & $14.7(0.5)$ & $17.2(0.6)$ & $39.4(0.9)$ & $35.2(0.7)$ & $83.6(0.4)$ & $21.7(2.2)$ \\
\hline Medium (8-17.5/day) & $16.7(0.6)$ & $18.5(0.6)$ & $40.0(0.8)$ & $35.5(0.7)$ & $83.3(0.4)$ & $19.5(1.9)$ \\
\hline High $(>17.5 /$ day $)$ & $16.8(0.6)$ & $18.1(0.6)$ & $39.3(0.9)$ & $35.2(0.7)$ & $84.1(0.4)$ & $14.6(1.5)$ \\
\hline $\mathrm{p}$ for trend & 0.008 & 0.35 & 0.94 & 0.95 & 0.42 & 0.008 \\
\hline \multicolumn{7}{|l|}{ Soy } \\
\hline Low (0/week) & $15.7(0.5)$ & $17.5(0.5)$ & $34.9(0.6)$ & $39.2(0.7)$ & $83.6(0.4)$ & $20.3(1.8)$ \\
\hline Medium (1-2.5/week) & $16.6(0.7)$ & $18.1(0.8)$ & $35.8(0.9)$ & $40.0(1.0)$ & $84.5(0.5)$ & $16.4(2.0)$ \\
\hline High $(\geq 2.5 /$ week $)$ & $16.0(0.6)$ & $18.3(0.6)$ & $35.5(0.7)$ & $40.0(0.8)$ & $83.3(0.4)$ & $17.3(1.7)$ \\
\hline $\mathrm{p}$ for trend & 0.94 & 0.66 & 0.56 & 0.66 & 0.83 & 0.22 \\
\hline \multicolumn{7}{|l|}{ Citrus } \\
\hline Low $(<1 /$ week $)$ & $15.9(0.6)$ & $18.1(0.7)$ & $34.3(0.8)$ & $38.5(0.9)$ & $83.8(0.4)$ & $16.7(1.7)$ \\
\hline Medium (1-4/week) & $15.9(0.5)$ & $17.6(0.6)$ & $35.7(0.7)$ & $40.1(0.8)$ & $83.9(0.4)$ & $18.5(1.8)$ \\
\hline High ( $\geq 5 /$ week) & $16.2(0.5)$ & $18.1(0.6)$ & $35.7(0.7)$ & $40.0(0.8)$ & $83.3(0.4)$ & $19.6(1.9)$ \\
\hline $\mathrm{p}$ for trend & 0.88 & 0.26 & 0.79 & 0.28 & 0.49 & 0.20 \\
\hline \multicolumn{7}{|l|}{ Crucifers } \\
\hline Low $(<1 /$ week $)$ & $15.2(0.5)$ & $16.9(0.6)$ & $34.4(0.8)$ & $38.6(0.9)$ & $84.8(0.4)$ & $20.1(2.1)$ \\
\hline Medium (1-2.5/week) & $16.0(0.5)$ & $18.5(0.6)$ & $36.0(0.7)$ & $40.5(0.8)$ & $83.0(0.4)$ & $20.5(1.9)$ \\
\hline High $(\geq 2.5 /$ week $)$ & $16.7(0.5)$ & $18.3(0.6)$ & $35.4(0.7)$ & $40.0(0.8)$ & $83.7(0.4)$ & $15.2(1.4)$ \\
\hline $\mathrm{p}$ for trend & 0.05 & 0.30 & 0.45 & 0.60 & 0.61 & 0.05 \\
\hline
\end{tabular}

All statistical analyses were performed on log-transformed percents of metabolite recovered (metabolite/total recovered $\times 100$ ) adjusted for calories, BMI, age, UGT1A6 genotype, sex, ethnicity, and urinary volume and $\mathrm{pH}$ (concentrations do not total $100 \%$ due to adjustment).

been attributed to hormonal changes during the menstrual cycle, percent body fat, glomerular filtration rate, and differences in biotransformation enzyme activity [26-28].

In the present analysis, Caucasians excreted statistically significantly more SUA and SUAPG than African-Americans and Asians. Although numerous studies have reported differences in drug metabolism among ethnic groups [29], few studies have looked at the effects of ethnicity on ASA metabolism specifically. Williams et al. [30] reported significantly lower plasma esterase activity (hydrolysis of ASA to SA) in individuals from Ghana compared to individuals living in the UK. Another group observed significant differences in SUA and several other pharmacokinetic parameters between two ethnic groups in Mexico [4]. Finally, in a twin study, Furst et al. [29] reported that variations in excretion rates of SA and SUA were significantly smaller between identical twins than between fraternal twins, suggesting genetic influences in metabolism.

The conversion of SA to SUA is achieved by glycine $N$-acyltransferase after activation to a coenzyme A (CoA) thioester by acyl-CoA synthetase [31]. Although a number of studies have observed increased SUA production with continued ASA administration [2], formal study of glycine $\mathrm{N}$-acyltransferase is largely non-existent. Only one study has looked at potential polymorphisms in the gene. Yamamoto et al. [32] identified 5 single nucleotide polymorphisms (SNPs) in the coding region and the exon-intron junctions of the glycine $\mathrm{N}$-acyltransferase gene among 95 Japanese individuals. The non-synonymous SNP in exon 5 was not present among 31 Caucasians, suggesting ethnic differences; however, allelic frequencies of all SNPs were low $(<3 \%)$, and the sample size was small. Whether these or other yet-to- 
be-described SNPs result in functional changes in enzyme activity is not known, but may be a potential explanation for our observed differences in SUA excretion among ethnicities.

Availability of glycine or CoA may also contribute to the variation in glycine conjugation and subsequent formation of SUA. Levy et al. [33] showed that glycine conjugation of salicylates becomes capacity limited in the therapeutic dose range. Work by Gregus et al. [34] suggested that this capacity-limited conjugation may be due to depletion of available glycine and CoA for the conjugation process. They demonstrated that administration of benzoic acid to rats, which is almost exclusively excreted as benzoylglycine, led to dose-dependent decreases in the rate of benzoic acid elimination [34]. In humans, inhibition of SUA formation was observed with co-administration of ASA with xylene, compared to ASA administration alone [35]. It is unlikely, however, that substrate depletion contributed greatly to the differences that we observed in our study given the administration of a single-dose of $600 \mathrm{mg}$ ASA. However, it is possible that regular exposure to xenobiotics or other factors may modulate glycine $\mathrm{N}$-acyltransferase enzyme activity, either through competitive inhibition or enzyme induction, thus playing a role in the variability observed in ASA metabolism.

Formation of SA glucuronides by UGTs provides alternate pathways to the capacitylimited glycine conjugation of SA to form SUA. We hypothesized that the ${ }^{*} 2$ variant in the UGT1A6 gene, which has been shown to result in slower metabolism of many phenolic substrates, would lead to reduced glucuronidation and clearance of SA, thought to be the major pharmacologically active compound in ASA [15]. However, contrary to our hypothesis, urinary excretion of ASA and its major metabolites was not altered by UGT1A6 genotype. The reason we did not observe any UGT1A6 genotype effects in this study may be because measurement of urinary excretion following a single, standard dose of ASA is not sensitive enough to detect differential UGT enzyme activities. Additionally, there may be compensation by other UGT isoforms for enzyme variants that result in decreased activity. When this project was conceived, the UGT1A6*2 variant reportedly exhibited slower metabolism of several phenolic substrates in vitro [8]. However, subsequent in vitro studies demonstrated enhanced glucuronidation for the homozygous variant $U G T 1 A 6^{*} 2$ genotype relative to the wild type for some substrates $[11,12]$. Two recent small-scale in vivo studies conducted in humans ( $\mathrm{n}=9$ and 28) evaluated the effects of UGT1A6 genotypes on ASA metabolism specifically, and also supported more rapid glucuronidation among homozygous carriers of $U G T 1 A 6^{*} 2$ $[5,6]$. Our results, in this sample of 528 , do not support either faster or slower metabolism of ASA among individuals with a UGT1A6*2 genotype.

We also evaluated the effects of dietary factors on ASA metabolism, particularly in foods containing bioactive phytochemicals known to induce UGT enzyme expression. We anticipated that individuals with the highest intakes of citrus, soy, and crucifers would have the greatest urinary excretion of glucuronides (SAAG and SAPG). There were no differences in urinary excretion of any metabolites with consumption of citrus or soy, or combined consumption of soy, citrus and cruciferous vegetables. Further, although higher servings of total vegetables, and crucifers in particular, were associated with significantly more urinary excretion of ASA and less SUAPG, excretion of SAAG and SAPG were not altered significantly. Our results, therefore, do not support increased glucuronidation activity among individuals with higher fruit, vegetable, or soy intake. The pattern of excretion does, however, suggest an inhibitory role for increased consumption of vegetables ( $>2.5$ servings/day) in general, and perhaps crucifers specifically, on glycine conjugation.

To our knowledge, this is the largest study to evaluate differences in ASA metabolism in healthy individuals, and the first to assess the relationship between UGT polymorphisms and the effects of dietary factors on the metabolism of ASA. Participants were carefully screened, selected on the basis of UGT1A6 genotype, and a number of inclusion and exclusion criteria ensured a relatively homogeneous study population. Nevertheless, there are several limita- 
tions. First, we collected only 8-hour urine, which may not completely reflect UGT activity on ASA metabolism in the body. However, we reported previously that the most substantial differences between $U G T 1 A 6^{*} 1 /{ }^{*} 1$ and ${ }^{*} 2 /{ }^{*} 2$ in ASA metabolite excretion showed up within the first $8 \mathrm{~h}$ [6]. Therefore, $8 \mathrm{~h}$ should be sufficient to observe differences in urinary metabolite excretion of ASA. Second, this study included only two of all the UGTs as indicators of in vivo glucuronidation activities. Human UGTs include UGT1A, UGT2A, UGT2B, UGT3 and UGT8 enzyme families, and many of the UGTs are highly polymorphic [36, 37]. We have shown previously that UGT 1A1, 1A3, 1A6, 1A7, 1A8, 1A9, 1A10, 2B4 and 2B7 Supersomes could all catalyze SAAG and SAPG glucuronidation in the ASA metabolic pathways in vitro [37-39]. However, only UGT1A6 has been associated with differential effects of ASA in population-based studies $[5,14,40,41]$. A haplotyping method evaluating all of the UGTs involved in ASA conjugation might help elucidate the specific contribution of individual UGTs, but this will require a substantially larger sample size. Third, we relied on an FFQ to capture usual dietary intake; FFQs are subject to lack of precision and inaccurate recall of dietary intake [42]. Although FFQs are less accurate in measuring vitamin or micronutrient intake, they provide a good measure of overall relative trends in consumption of specific food groups. However, the narrow range of intake, particularly for soy and crucifers, may have reduced our ability to detect modifying effects.

In conclusion, of the factors evaluated, ethnicity and sex were the greatest, albeit modest, contributors to the variability in ASA metabolism, and UGT1A6 was not a major factor. Diet may influence pharmacokinetic parameters of ASA, but the effects appear to be more through modulation of glycine conjugation than via glucuronidation. Given the differences in metabolism by ethnicity, future studies should include evaluation of other enzymes, including glycine- $N$-acyltransferase and polymorphisms in other UGT family members.

\section{Acknowledgment}

This work was supported by grants R01 CA94954, R01 CA92288, and R25 CA94880 from the National Institutes of Health, National Cancer Institute.

\section{References}

1 Court M: Interindividual variability in hepatic drug glucuronidation: studies into the role of age, sex, enzyme inducers, and genetic polymorphism using the human liver bank as a model system. Drug Metab Rev 2010;42:202-217.

2 Needs CJ, Brooks PM: Clinical pharmacokinetics of the salicylates. Clin Pharmacokinet 1985;10:164-177.

- 3 Miaskiewicz SL, Shively CA, Vesell ES: Sex differences in absorption kinetics of sodium salicylate. Clin Pharmacol Ther 1982;31:30-37.

-4 Lares-Asseff I, Juarez-Olguin H, Flores-Perez J, Guille-Perez A, Vargas A: Pharmacokinetics and metabolic rates of acetyl salicylic acid and its metabolites in an Otomi ethnic group of Mexico. Biol Pharm Bull 2004;27:706-709.

5 van Oijen M: Effect of genetic polymorphisms in UDP-glucuronosyltransferase 1A6 (UGT1A6) on acetylsalicylic acid metabolism in healthy female volunteers. Pharmacology 2009;83:237-242.

6 Chen Y, Kuehl GE, Bigler J, Rimorin CF, Schwarz Y, Shen DD, et al: UGT1A6 polymorphism and salicylic acid glucuronidation following aspirin. Pharmacogenet Genomics 2007;17:571-579.

7 Hutt AJ, Caldwell J, Smith RL: The metabolism of aspirin in man: a population study. Xenobiotica 1986;16:239-249.

8 Ciotti M, Marrone A, Potter C, Owens IS: Genetic polymorphism in the human UGT1A6 (planar phenol) UDP-glucuronosyltransferase: pharmacological implications. Pharmacogenetics 1997;7:485-495.

-9 Bosma P, Chowdhury JR, Bakker C, Gantla S, de Boer N, Oostra BA, et al: The genetic basis of the reduced expression of bilirubin UDP-glucuronosyltransferase 1 in Gilbert's syndrome. N Engl J Med 1995;333:1171-1175.

10 Guillemette C, Millikan RC, Newman B, Housman DE: Genetic polymorphisms in uridine diphospho-glucuronosyltransferase 1A1 and association with breast cancer among African Americans. Cancer Res 2000;60:950-956.

- 11 Nagar S, Zalatoris JJ, Blanchard RL: Human UGT1A6 pharmacogenetics: identification of a novel SNP, characterization of allele frequencies and functional analysis of recombinant allozymes in human liver tissue and in cultured cells. Pharmacogenetics 2004; 14:487-499. 


\section{Journal of \\ Nutrigenetics Nutrigenomics}

J Nutrigenet Nutrigenomics 2011;4:110-118

DOI: $10.1159 / 000327782$

Published online: May 28, 2011

2011 S. Karger AG, Basel

Navarro et al.: Determinants of Aspirin Metabolism

-12 Krishnaswamy S, Hao Q, Al-Rohaimi A, Hesse LM, von Moltke LL, Greenblatt DJ, et al: UDP glucuronosyltransferase (UGT) 1A6 pharmacogenetics: II. Functional impact of the three most common nonsynonymous UGT1A6 polymorphisms (S7A, T181A, and R184S). J Pharmacol Exp Ther 2005;313:1340-1346.

13 Lampe JW, Bigler J, Horner NK, Potter JD: UDP-glucuronosyltransferase (UGT1A1*28 and UGT1A6*2) polymorphisms in Caucasians and Asians: relationships to serum bilirubin concentrations. Pharmacogenetics 1999;9:341-349.

14 Bigler J, Whitton J, Lampe JW, Fosdick L, Bostick RM, Potter JD: CYP2C9 and UGT1A6 genotypes modulate the protective effect of aspirin on colon adenoma risk. Cancer Res 2001;61:3566-3569.

15 Levine RR: Pharmacology: Drug Actions and Reactions, ed 6. New York, Parthenon, 2000.

- 16 Saracino MR, Bigler J, Schwarz Y, Chang JL, Li S, Li L, et al: Citrus fruit intake is associated with lower serum bilirubin concentration among women with the UGT1A1*28 polymorphism. J Nutr 2009;139:555-560.

$\checkmark 17$ Navarro SL, Peterson S, Chen C, Makar KW, Schwarz Y, King IB, et al: Cruciferous vegetable feeding alters UGT1A1 activity: diet- and genotype-dependent changes in serum bilirubin in a controlled feeding trial. Cancer Prev Res 2009; 2:345-352.

18 Saracino MR, Lampe JW: Phytochemical regulation of UDP-glucuronosyltransferases: implications for cancer prevention. Nutr Cancer 2007;59:121-141.

19 Dinkova-Kostova AT, Holtzclaw WD, Wakabayashi N: Keap1, the sensor for electrophiles and oxidants that regulates the phase 2 response, is a zinc metalloprotein. Biochemistry 2005;44:6889-6899.

20 Lampe JW, Peterson S: Brassica, biotransformation and cancer risk: genetic polymorphisms alter the preventive effects of cruciferous vegetables. J Nutr 2002;132:2991-2994.

21 Nho CW, Jeffery E: The synergistic upregulation of phase II detoxification enzymes by glucosinolate breakdown products in cruciferous vegetables. Toxicol Appl Pharmacol 2001;174:146-152.

22 Willett W: Nutritional Epidemiology, ed 2. New York, Oxford University Press, 1998.

23 Liu JH, Smith PC: Direct analysis of salicylic acid, salicyl acyl glucuronide, salicyluric acid and gentisic acid in human plasma and urine by high-performance liquid chromatography. J Chromatogr B Biomed Appl 1996;675:61-70.

- 24 Ho KY, Evans WS, Blizzard RM, Veldhuis JD, Merriam GR, Samojlik E, et al: Effects of sex and age on the 24-hour profile of growth hormone secretion in man: importance of endogenous estradiol concentrations. J Clin Endocrinol Metab 1987;64:51-58.

25 Miners JO, Grgurinovich N, Whitehead AG, Robson RA, Birkett DJ: Influence of gender and oral contraceptive steroids on the metabolism of salicylic acid and acetylsalicylic acid. Br J Clin Pharmacol 1986;22:135-142.

26 Anderson GD: Sex differences in drug metabolism: cytochrome P-450 and uridine diphosphate glucuronosyltransferase. J Gend Specif Med 2002;5:25-33.

-27 Gupta KC, Joshi JV, Hazari K, Pohujani SM, Satoskar RS: Effect of low estrogen combination oral contraceptive on metabolism of aspirin and phenylbutazone. Int J Clin Pharmacol Ther Toxicol 1982;20:511-513.

28 Anderson GD: Gender differences in pharmacological response. Int Rev Neurobiol 2008;83:1-10.

29 Furst DE, Gupta N, Paulus HE: Salicylate metabolism in twins. Evidence suggesting a genetic influence and induction of salicylurate formation. J Clin Invest 1977;60:32-42.

- 30 Williams FM, Nicholson EN, Woolhouse NW, Adjepon-Yamoah KK, Rawlins MD: Activity of esterases in plasma from Ghanaian and British subjects. Eur J Clin Pharmacol 1986;31:485-489.

-31 Knights KM, Sykes MJ, Miners JO: Amino acid conjugation: contribution to the metabolism and toxicity of xenobiotic carboxylic acids. Expert Opin Drug Metab Toxicol 2007;3:159-168.

-32 Yamamoto A, Nonen S, Fukuda T, Yamazaki H, Azuma J: Genetic polymorphisms of glycine N-acyltransferase in Japanese individuals. Drug Metab Pharmacokinet 2009;24:114-117.

-33 Levy G, Tsuchiya T, Amsel LP: Limited capacity for salicyl phenolic glucuronide formation and its effect on the kinetics of salicylate elimination in man. Clin Pharmacol Ther 1972;13:258-268.

-34 Gregus Z, Fekete T, Varga F, Klaassen CD: Availability of glycine and coenzyme A limits glycine conjugation in vivo. Drug Metab Dispos 1992;20:234-240.

- 35 Campbell L, Wilson HK, Samuel AM, Gompertz D: Interactions of m-xylene and aspirin metabolism in man. Br J Ind Med 1988;45:127-132.

-36 Mackenzie PI, Bock KW, Burchell B, Guillemette C, Ikushiro S, Iyanagi T, et al: Nomenclature update for the mammalian UDP glycosyltransferase (UGT) gene superfamily. Pharmacogenet Genomics 2005;15:677-685.

37 Thomas SS, Li SS, Lampe JW, Potter JD, Bigler J: Genetic variability, haplotypes, and htSNPs for exons 1 at the human UGT1A locus. Hum Mutat 2006;27:717.

- 38 Kuehl GE, Lampe JW, Potter JD, Bigler J: Glucuronidation of nonsteroidal anti-inflammatory drugs: identifying the enzymes responsible in human liver microsomes. Drug Metab Dispos 2005;33:1027-1035.

- 39 Maruo Y, Iwai M, Mori A, Sato H, Takeuchi Y: Polymorphism of UDP-glucuronosyltransferase and drug metabolism. Curr Drug Metab 2005;6:91-99.

40 Chan AT, Tranah GJ, Giovannucci EL, Hunter DJ, Fuchs CS: Genetic variants in the UGT1A6 enzyme, aspirin use, and the risk of colorectal adenoma. J Natl Cancer Inst 2005;97:457-460.

-41 McGreavey LE, Turner F, Smith G, Boylan K, Timothy Bishop D, Forman D, et al: No evidence that polymorphisms in CYP2C8, CYP2C9, UGT1A6, PPARdelta and PPARgamma act as modifiers of the protective effect of regular NSAID use on the risk of colorectal carcinoma. Pharmacogenet Genomics 2005;15:713-721.

-42 Kristal AR, Vizenor NC, Patterson RE, Neuhouser ML, Shattuck AL, McLerran D: Precision and bias of food frequency-based measures of fruit and vegetable intakes. Cancer Epidemiol Biomarkers Prev 2000;9:939-944. 